\title{
Diastereoselective conjugate addition of $(R)$-4-phenyl-2-oxazolidinone to dialkyl alkylidenemalonates
}

\author{
Julien Capra, ${ }^{\mathrm{a}}$ Bo Gao, ${ }^{\mathrm{a}}$ Hélène Hemmery, ${ }^{\mathrm{a}}$ Pierre Thuéry, ${ }^{\mathrm{b}}$ and Thierry Le Gall ${ }^{\mathrm{a}, *}$ \\ ${ }^{a}$ CEA, iBiTecS, Service de Chimie Bioorganique et de Marquage, Bât. 547, 91191, Gif-sur-Yvette, \\ France \\ ${ }^{b}$ CEA, IRAMIS, NIMBE, UMR 3299 CEA/CNRS, Bât. 125, 91191 Gif-sur-Yvette, France \\ E-mail: thierry.legall@cea.fr
}

DOI: $\underline{\text { http://dx.doi.org/10.3998/ark.5550190.p008.925 }}$

\begin{abstract}
The addition of the potassium salt of $(R)$-4-phenyl-2-oxazolidinone to dialkyl alkylidenemalonates under various conditions is reported. Very good diastereoselectivities ( $>90 \% \mathrm{de}$ ) were obtained in several cases. Conversion of one of the adducts to the $\beta$-amino acid $(S)$ - $\beta$-leucine was achieved in two straightforward steps.
\end{abstract}

Keywords: Amino acids, asymmetric synthesis, malonates, nucleophilic addition, oxazolidinone

\section{Introduction}

$\beta$-Amino acids are components or precursors of many bioactive substances and thus methods for their asymmetric synthesis have been developed using various strategies. ${ }^{1-3}$ Among these, approaches involving an aza-Michael reaction have often been used. In this process, the asymmetric induction may come either from a chiral nitrogen nucleophile, ${ }^{4-6}$ or from a chiral conjugate electrophile, $^{7-11}$ or from a chiral ligand, employed either stoichiometrically ${ }^{12,13}$ or catalytically. ${ }^{14-16}$

We previously reported the highly diastereoselective conjugate addition of $(R)$ - and $(S)$ 4-phenyl-2-oxazolidinone to nitroalkenes. ${ }^{17}$ The corresponding adducts then served as precursors to enantiopure $\beta$-amino acids, ${ }^{18}$ 1,2-diamines, ${ }^{17,18}$ and amines, ${ }^{19}$ which were obtained after modification of the nitro function and cleavage of the oxazolidinone moiety to release an amine function. In this article, we report the results obtained in the conjugate addition of $(R)$-4-phenyl-2oxazolidinone to dialkyl alkylidenemalonates, leading to precursors of $\beta$-amino acids. 


\section{Results and Discussion}

Preliminary attempts to perform the conjugate addition of the potassium salt of 4-phenyl-2oxazolidinone 1 to alkenes activated by only one ester function, methyl crotonate and methyl cinnamate were unsuccessful. It should be noted that the conjugate addition of 2-oxazolidinone to methyl acrylate, catalyzed by either 1,8-diazabicyclo[5.4.0]undec-7-ene $(\mathrm{DBU})^{20}$ or tetramethylammonium fluoride ${ }^{21}$ has been reported. In our case, it was obvious that more activated electrophiles had to be employed, and dialkyl alkylidenemalonates $\mathbf{2}$ were then tested. Compounds 2 were obtained from commercial sources or prepared from dimethyl malonate, according to reported procedures, ${ }^{22,23}$ and several conjugate additions were then performed (Scheme 1).

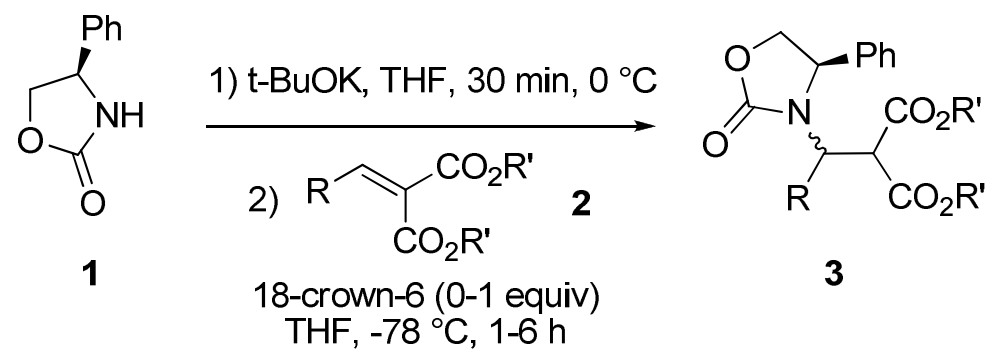

Scheme 1. Conjugate addition of $(R)$-4-phenyl-2-oxazolidinone 1 to dialkyl alkylidenemalonates.

The potassium salt of oxazolidinone 1 was generated in THF at $0{ }^{\circ} \mathrm{C}$ by addition of potassium tert-butoxide, either in the presence of 18 -crown- 6 or not. After $30 \mathrm{~min}$ at $0{ }^{\circ} \mathrm{C}$, the suspension obtained was cooled at $-78{ }^{\circ} \mathrm{C}$, and a solution of dialkyl alkylidenemalonate in THF was added. In the first experiments, the temperature was allowed to reach room temperature; however, in these conditions, no adduct was isolated, which was attributed to a retroaddition at room temperature. Three different conditions were then employed in the other experiments: The reaction mixture was stirred at $-78{ }^{\circ} \mathrm{C}$ for $1-6 \mathrm{~h}$ and then hydrolyzed (method $\mathrm{A}$ ); or the reaction mixture was stirred at $-78{ }^{\circ} \mathrm{C}$ for $1 \mathrm{~h}$, then stirred at $-20{ }^{\circ} \mathrm{C}$ for $1 \mathrm{~h}(\operatorname{method} \mathrm{B})$ or for $12 \mathrm{~h}(\operatorname{method} \mathrm{C})$ and then hydrolyzed (Table 1).

Unlike the conjugated mono-esters, most tested dialkyl alkylidenemalonates reacted with the salt of oxazolidinone 1, and several reactions proceeded with diastereomeric excesses (de) of $90 \%$ or more. The diastereoselectivity depended on the nature of the $\mathrm{R}$ group. Hence, good diastereoisomeric excesses were obtained starting from compounds 2 where $\mathrm{R}=$ isopropyl, cyclohexyl and phenyl, but not from compound 2a, where $\mathrm{R}=$ methyl, a smaller group. This suggested that the diastereofacial selectivity improved when the $\mathrm{R}$ group was more sterically demanding. However, no reaction was observed starting from a malonate bearing the more crowded tert-butyl group (entry 8).

In several cases where the reaction mixture was maintained at $-78{ }^{\circ} \mathrm{C}$, the yields were poor, owing to incomplete additions (entries 1,9 and 11). Adding one equivalent of 18-crown-6 improved the yield, but had a negative impact on the diastereoselectivity (entry 2). The yield was usually 
better when the temperature was raised to $-20{ }^{\circ} \mathrm{C}$ prior to the addition of water. However, the reaction was still incomplete after $12 \mathrm{~h}$ in some cases (entries 3 and 4).

Table 1. Preparation of oxazolidinones 3

\begin{tabular}{llllllll}
\hline Entry & & $\mathrm{R}$ & $\mathrm{R}{ }^{\prime}$ & Product $^{\text {18-Crown-6 }}$ & $\begin{array}{c}\text { 18 } \\
\text { (equiv) }\end{array}$ & $D e^{d}$ & Yield (\%) \\
\hline 1 & $\mathrm{Me}$ & $\mathrm{Et}$ & $\mathbf{3 a}^{a}$ & 0 & 36 & 50 \\
2 & $\mathrm{Me}$ & $\mathrm{Et}$ & $\mathbf{3 a}^{a}$ & 1 & 28 & 99 \\
3 & $\mathrm{Et}$ & $\mathrm{Me}$ & $\mathbf{3 b}^{c}$ & 0 & 67 & 37 \\
4 & $\mathrm{Mentyl}$ & $\mathrm{Me}$ & $\mathbf{3 c}^{c}$ & 0 & 45 & 43 \\
5 & $\mathrm{iBu}$ & $\mathrm{Me}$ & $\mathbf{3 d}^{c}$ & 0 & 41 & 65 \\
6 & $i \mathrm{Pr}$ & $\mathrm{Me}$ & $\mathbf{3 e}^{b}$ & 0 & 91 & 60 \\
7 & $i \mathrm{Pr}$ & $\mathrm{Me}$ & $\mathbf{3 e}^{c}$ & 0 & 92 & 63 \\
8 & $t \mathrm{Bu}$ & $\mathrm{Me}$ & $\mathbf{3 f}^{b}$ & 1 & - & 0 \\
9 & $\mathrm{Melohexyl}$ & $\mathrm{Me}$ & $\mathbf{3 g}^{a}$ & 0 & 94 & 21 \\
10 & $\mathrm{Myclohexyl}$ & $\mathrm{Me}$ & $\mathbf{3 g}^{b}$ & 1 & 46 & 77 \\
11 & $\mathrm{Ph}$ & $\mathrm{Me}$ & $\mathbf{3 h}^{a}$ & 0 & 98 & 30 \\
12 & $\mathrm{Ph}$ & $\mathrm{Me}$ & $\mathbf{3 h}^{b}$ & 1 & 90 & 70 \\
\hline
\end{tabular}

${ }^{a}$ Method A (see text). ${ }^{b}$ Method B (see text). ${ }^{c}$ Method C (see text). ${ }^{d}$ Diastereomeric excess determined by ${ }^{1} \mathrm{H}$ NMR.

The major diastereoisomer of adduct $\mathbf{3 h}$, obtained from $(R)$-4-phenyl-2-oxazolidinone and dimethyl benzylidenemalonate was isolated by crystallization and single crystal X-ray diffraction studies established the configuration of the newly created stereogenic center as $S$ (Figure 1).

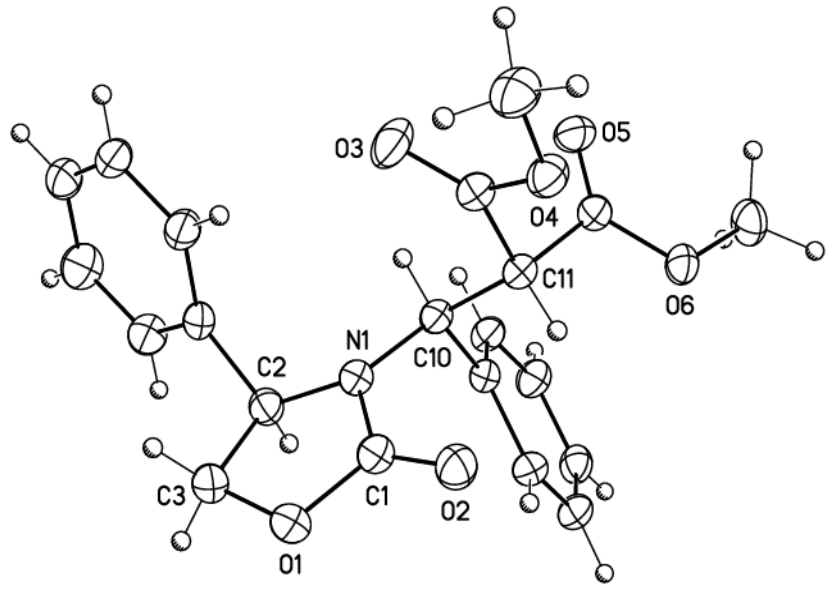

Figure 1. Molecular structure of the major diastereoisomer of adduct $\mathbf{3 h}$. Displacement ellipsoids are drawn at the $30 \%$ probability level. 
Adducts 3 are potential precursors to the corresponding $\beta$-amino acids, and it is worthy of note that oxazolidinone esters have been previously used as intermediates in the stereoselective syntheses of $\beta$-amino acids. ${ }^{24,25}$ The two steps needed to obtain a $\beta$-amino acid from an adduct 3 are firstly the conversion of the diester moiety to a single carboxylic acid via an acidic hydrolysis followed by a decarboxylation, and secondly the cleavage of the oxazolidinone moiety leading to an amine function.

As an example of such transformation, compound $\mathbf{3 e}$ was efficiently converted to the $\beta$-amino acid 5 in two steps. Thus, $3 \mathbf{e}$ was first heated in $6 \mathrm{~N} \mathrm{HCl}$ for $15 \mathrm{~h}$ at $100{ }^{\circ} \mathrm{C},{ }^{26}$ leading to the acid 4 in $53 \%$ yield, after two recrystallizations from diethyl ether. It should be noted that an attempt to hydrolyze the ester functions under basic conditions $\left(\mathrm{LiOH}, \mathrm{THF} / \mathrm{H}_{2} \mathrm{O}\right)$ resulted in retroaddition leading to oxazolidinone 1 . The cleavage of the oxazolidinone ring was then cleanly realized by treating 4 with lithium in liquid ammonia, ${ }^{27,28}$ affording 3 -amino-4-methylpentanoic acid $(\beta$ leucine, 5) in $77 \%$ yield.

The specific rotations reported for various samples of $\beta$-leucine have been recently compiled. ${ }^{5}$ The specific rotation of $5\left([\alpha]_{\mathrm{D}}{ }^{20}-49.6\right.$. $c 0.4$, water) was in accord with those reported for the $S$ isomer [lit. ${ }^{5}-53$ ( $c$ 2.0, water)]. Thus the absolute chemistry of the stereogenic center created in the conjugate addition step is $S$, and the ee is $93 \%$.
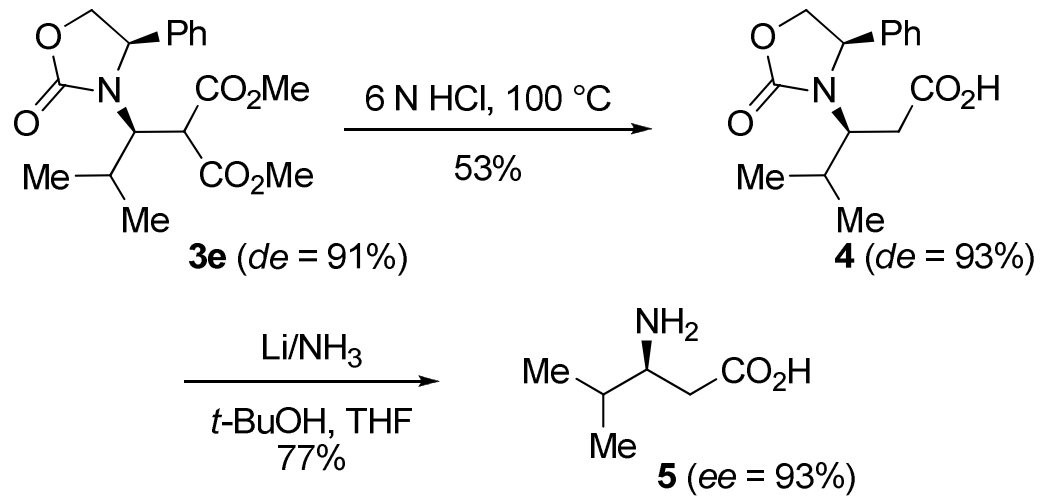

Scheme 2. Two-step conversion of adduct 3e to $(S)$ - $\beta$-leucine (5).

A postulated 8-membered transition state leading to the observed major isomer is indicated in the Scheme 3. It involves an attack on the $R e$ face of the electrophile. The side chain group of the alkylidenemalonate is in a pseudo-equatorial position, which takes into account the fact that the selectivity was enhanced as the side chain group was bulkier. Similar transition states have been postulated for reactions of oxazolidinone anions with other electrophiles. ${ }^{29,30}$ 


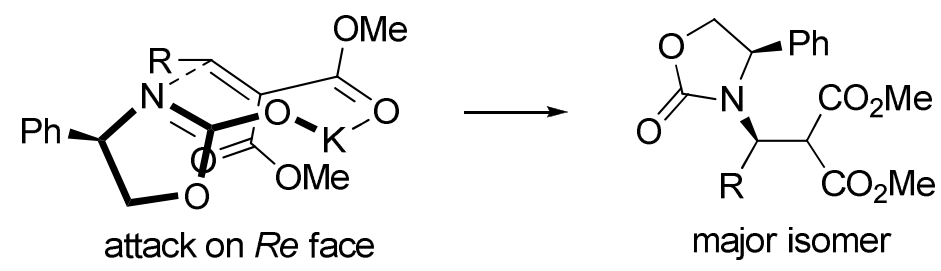

Scheme 3. Postulated transition state for the formation of the major adduct.

\section{Conclusions}

In summary, the potassium salt of $(R)$-4-phenyl-2-oxazolidinone reacts with dialkyl alkylidenemalonates to afford the corresponding conjugate addition products. The diastereoselectivity of the reaction was better for electrophiles having more sterically demanding side chain groups, and diastereomeric excesses of $90 \%$ or more were obtained in several cases; although an electrophile bearing a bulky tert-butyl side chain was unreactive. The adduct obtained from the reaction involving dimethyl 2-(2-methylpropylidene)malonate was converted in two steps to the amino acid $(S)$ - $\beta$-leucine, thus showing that the method permits a valuable enantioselective synthesis of the biologically important $\beta$-amino acids.

\section{Experimental Section}

General. ${ }^{1} \mathrm{H}$ and ${ }^{13} \mathrm{C}$ NMR spectra were recorded on a Bruker Advanced 400 spectrometer and chemical shifts are reported in ppm downfield from TMS for ${ }^{1} \mathrm{H}$ and ${ }^{13} \mathrm{C}$ NMR. IR spectra were recorded on a Perkin Elmer System 2000 FT-IR (liquid films or KBr pellets for solids). MS were recorded on a Mariner ESI TOF (Perseptive Biosystems) mass spectrometer. Mps were determined on a Büchi B-540 apparatus. HRMS were performed at the Service de Spectrométrie de Masse, ICSN, CNRS, Gif-sur-Yvette. Chromatographies were carried out using a CombiFlash system (Teledyne ISCO). TLCs were developed on silica gel $60 \mathrm{~F}_{254}$ plates, with detection by UV light and by an ethanol solution of phosphomolybdic acid. THF was freshly distilled on sodiumbenzophenone.

General procedure for the conjugate addition of $(R)-4$-phenyl-2-oxazolidinone to dialkyl alkylidenemalonates. $(R)$-4-Phenyloxazolidin-2-one $(1.0 \mathrm{mmol})$ and potassium tert-butoxide $(1.0$ $\mathrm{mmol})$, were placed in a flask under argon atmosphere, cooled at $0{ }^{\circ} \mathrm{C}$. THF $(8 \mathrm{~mL})$ was added and the white suspension obtained was stirred at $0{ }^{\circ} \mathrm{C}$ for $30 \mathrm{~min}$ and then cooled to $-78{ }^{\circ} \mathrm{C}$. A solution of the dialkyl alkylidenemalonate $2(1.0 \mathrm{mmol})$ in THF $(2 \mathrm{~mL})$ cooled at $0{ }^{\circ} \mathrm{C}$ was added slowly and then the reaction mixture was stirred at $-78{ }^{\circ} \mathrm{C}$ for $1-6 \mathrm{~h}$. A saturated aqueous $\mathrm{NH}_{4} \mathrm{Cl}$ solution $(5 \mathrm{~mL})$ was added. The aqueous phase was extracted with diethyl ether $(3 \times 10 \mathrm{~mL})$. The combined 
organic layers were washed with brine $(10 \mathrm{~mL})$, dried $\left(\mathrm{MgSO}_{4}\right)$, filtered and concentrated under vacuum. The residue obtained was then purified by silica gel chromatography (cyclohexane/EtOAc) to afford the adduct 3.

Diethyl 2-\{1-[(R)-2-oxo-4-phenyloxazolidin-3-yl]ethyl $\}$ malonate (3a). This compound was obtained by the General Procedure (see Table 1, entry 2). Yield: 99\%. 28\% de. Colorless oil. IR (film) $v_{\max } 3055,2987,1754(\mathrm{C}=\mathrm{O}), 1458,1421,1265,1035,896,738,705 \mathrm{~cm}^{-1}$. ${ }^{1} \mathrm{H}$ NMR (400 $\mathrm{MHz}_{\mathrm{CDCl}}$ ): $\delta$ (major isomer) 7.45-7.30 (m, 5H, Ar H), 4.87 (dd, $J$ 8.7, $5.7 \mathrm{~Hz}, 1 \mathrm{H}, \mathrm{NCHPh}$ ), $4.55(\mathrm{t}, J 8.7 \mathrm{~Hz}, 1 \mathrm{H}, \mathrm{CHHCHPh}), 4.42\left(\mathrm{dq}, J\right.$ 9.9, $\left.6.9 \mathrm{~Hz}, 1 \mathrm{H}, \mathrm{CH}_{3} \mathrm{CHN}\right), 4.32-4.10(\mathrm{~m}, 5 \mathrm{H}$, $\mathrm{CH} H \mathrm{CHPh}$ and $\left.\mathrm{OCH}_{2} \mathrm{CH}_{3}\right), 3.92\left(\mathrm{~d}, J 9.9 \mathrm{~Hz}, 1 \mathrm{H}, \mathrm{CHCO}_{2}\right), 1.35-1.20\left(\mathrm{~m}, 6 \mathrm{H}, \mathrm{CH}_{2} \mathrm{CH}_{3}\right), 0.98(\mathrm{~d}, J$ $6.9 \mathrm{~Hz}, 3 \mathrm{H}, \mathrm{CHCH}_{3}$ ) ppm; $\delta$ (minor isomer) 7.45-7.30 (m, 5H, $\left.\mathrm{Ar} H\right), 4.91(\mathrm{dd}, J 9.0,6.5 \mathrm{~Hz}, 1 \mathrm{H}$, $\mathrm{NCHPh}), 4.55(\mathrm{t}, J 9.0 \mathrm{~Hz}, 1 \mathrm{H}, \mathrm{CHHCHPh}), 4.32-4.10\left(\mathrm{~m}, 6 \mathrm{H}, \mathrm{CH} H \mathrm{CHPh}, \mathrm{OCH}_{2} \mathrm{CH}_{3}\right.$ and $\mathrm{CHCO}_{2}$ ), $3.74\left(\mathrm{dq}, J 10.9,6.9 \mathrm{~Hz}, 1 \mathrm{H}, \mathrm{CH}_{3} \mathrm{CHN}\right), 1.35-1.20\left(\mathrm{~m}, 6 \mathrm{H}, \mathrm{CH}_{2} \mathrm{CH}_{3}\right), 1.18(\mathrm{~d}, J 6.9 \mathrm{~Hz}$, $\left.3 \mathrm{H}, \mathrm{CHCH}_{3}\right)$ ppm. ${ }^{13} \mathrm{C} \mathrm{NMR}\left(100 \mathrm{MHz}, \mathrm{CDCl}_{3}\right): \delta$ (two isomers) $167.6,167.2,167.1,157.9$, 156.6, 139.5, 138.4, 129.3, 129.2, 129.1, 127.8, 127.1, 70.6, 70.2, 62.5, 61.9, 61.7, 61.7, 58.7, 55.6, 54.6, 49.6, 49.5, 16.7, 16.4, 13.9, 13.8 ppm. HRMS (ESI): calcd. for $\mathrm{C}_{18} \mathrm{H}_{24} \mathrm{NO}_{6}[\mathrm{M}+\mathrm{H}]^{+}$ 350.1604 ; found 350.1605 .

Dimethyl 2-\{1-[(R)-2-oxo-4-phenyloxazolidin-3-yl]propyl $\}$ malonate (3b). This compound was obtained by the General Procedure (see Table 1, entry 3). Yield: 37\%. 67\% de. Colorless crystals; $\mathrm{mp} 80-83{ }^{\circ} \mathrm{C}$. IR (KBr pellet) $v_{\max } 2975,2955,2880,1757$ (C=O), 1458, 1434, 1413, 1321, 1271, 1219, 1197, 1151, 1062, 1037, 1018, 962, 922, 859, 771, 759, 713, $632 \mathrm{~cm}^{-1}$. ${ }^{1} \mathrm{H}$ NMR (400 MHz, $\left.\mathrm{CDCl}_{3}\right): \delta$ (major isomer) 7.45-7.35 (m, 5H, Ar $\left.H\right), 4.89(\mathrm{dd}, J 8.8,5.5 \mathrm{~Hz}, 1 \mathrm{H}, \mathrm{NCHPh}), 4.57(\mathrm{t}, J$ $8.8 \mathrm{~Hz}, 1 \mathrm{H}, \mathrm{CHHCHPh}), 4.30-4.23$ (m, 1H, NCHEt), 4.19 (dd, J 8.8, 5.5 Hz, 1H, CHHCHPh), 3.91 (d, J $\left.9.7 \mathrm{~Hz}, 1 \mathrm{H}, \mathrm{CHCO}_{2}\right), 3.72\left(\mathrm{~s}, 3 \mathrm{H}, \mathrm{OCH}_{3}\right), 3.70\left(\mathrm{~s}, 3 \mathrm{H}, \mathrm{OCH}_{3}\right), 1.42-1.34\left(\mathrm{~m}, 1 \mathrm{H}, \mathrm{CHHCH}_{3}\right)$, 1.24-1.17 (m, 1H, $\left.\mathrm{CHHCH}_{3}\right), 0.77\left(\mathrm{t}, J 7.2 \mathrm{~Hz}, 3 \mathrm{H}, \mathrm{CH}_{2} \mathrm{CH}_{3}\right)$ ppm. ${ }^{13} \mathrm{C} \mathrm{NMR}\left(100 \mathrm{MHz}, \mathrm{CDCl}_{3}\right): \delta$ (major isomer) $167.5\left(\mathrm{CO}_{2} \mathrm{Me}\right), 167.4\left(\mathrm{CO}_{2} \mathrm{Me}\right), 158.3(\mathrm{C}=\mathrm{O}), 139.3,129.0(2 \mathrm{C}), 128.3(2 \mathrm{C})$, 127.3, 70.6 (CH2OCO), 59.2 (CHEt), $\left.55.8(\mathrm{CHPh}), 52.7\left(\mathrm{CH}\left(\mathrm{CO}_{2} \mathrm{Et}\right)\right)_{2}\right), 52.7\left(\mathrm{OCH}_{3}\right), 52.6$ $\left(\mathrm{OCH}_{3}\right), 23.8\left(\mathrm{CH}_{2} \mathrm{CH}_{3}\right), 10.7\left(\mathrm{CH}_{3}\right)$ ppm. HRMS (ESI): calcd. for $\mathrm{C}_{19} \mathrm{H}_{26} \mathrm{NO}_{6}[\mathrm{M}+\mathrm{H}]^{+} 336.1447$; found 336.1456 .

Dimethyl 2-\{1-[(R)-2-oxo-4-phenyloxazolidin-3-yl]hexyl\}malonate (3c). This compound was obtained by the General Procedure (see Table 1, entry 4). Yield: $43 \% .45 \%$ de. Colorless oil. IR (film) $\nu_{\max } 2955,2860,1754(\mathrm{C}=\mathrm{O}), 1459,1435,1411,1222,1158,1042,762,735,704 \mathrm{~cm}^{-1} .{ }^{1} \mathrm{H}$ NMR (400 MHz, $\mathrm{CDCl}_{3}$ ): $\delta$ (major isomer) 7.45-7.30 (m, 5H, Ar-H), $4.87(\mathrm{dd}, J 8.7,5.0 \mathrm{~Hz}, 1 \mathrm{H}$, $\mathrm{NCHPh}), 4.56$ (t, $J 8.7 \mathrm{~Hz}, 1 \mathrm{H}, \mathrm{CHHCHPh}), 4.38\left(\mathrm{~m}, 1 \mathrm{H}, \mathrm{NCHC}_{5} \mathrm{H}_{11}\right), 4.19$ (dd, J 8.7, $5.0 \mathrm{~Hz}, 1 \mathrm{H}$, $\mathrm{CH} H \mathrm{CHPh}), 3.84\left(\mathrm{~d}, J 9.9 \mathrm{~Hz}, 1 \mathrm{H}, \mathrm{CHCO}_{2}\right), 3.72\left(\mathrm{~s}, 3 \mathrm{H}, \mathrm{OCH}_{3}\right), 3.71\left(\mathrm{~s}, 3 \mathrm{H}, \mathrm{OCH}_{3}\right), 1.30-0.90$ $\left(\mathrm{m}, 8 \mathrm{H}, \mathrm{CH}_{2}\right), 0.76\left(\mathrm{t}, J 7.4 \mathrm{~Hz}, 3 \mathrm{H}, \mathrm{CH}_{2} \mathrm{CH}_{3}\right) \mathrm{ppm} .{ }^{13} \mathrm{C} \mathrm{NMR}\left(100 \mathrm{MHz}, \mathrm{CDCl}_{3}\right): \delta$ (major isomer) $167.5\left(2 \mathrm{CO}_{2} \mathrm{Me}\right), 158.4(\mathrm{C}=\mathrm{O}), 139.7,129.0$ (2C), 128.3 (2C), 127.2, $70.7\left(\mathrm{CH}_{2} \mathrm{OCO}\right), 58.7$ $\left(\mathrm{CHC}_{5} \mathrm{H}_{11}\right), 54.7(\mathrm{CHPh}), 54.5\left(\mathrm{CH}\left(\mathrm{CO}_{2} \mathrm{Et}\right)_{2}\right), 52.7\left(\mathrm{OCH}_{3}\right), 52.6\left(\mathrm{OCH}_{3}\right), 31.0,30.7,25.8,22.2$, $13.8\left(\mathrm{CH}_{3}\right)$ ppm. HRMS (ESI): calcd. for $\mathrm{C}_{20} \mathrm{H}_{28} \mathrm{NO}_{6}[\mathrm{M}+\mathrm{H}]^{+}$378.1917. found 378.1899.

Dimethyl 2-\{3-methyl-1-[(R)-2-oxo-4-phenyloxazolidin-3-yl]butyl\}malonate (3d). This compound was obtained by the General Procedure (see Table 1, entry 5). Yield: 65\%; 41\% de. 
Colorless oil. IR (film) $v_{\max } 3055,2987,2930,2306,1754(\mathrm{C}=\mathrm{O}), 1422,1265,896,738,705 \mathrm{~cm}^{-1}$. ${ }^{1} \mathrm{H} \mathrm{NMR}\left(400 \mathrm{MHz}, \mathrm{CDCl}_{3}\right.$ ): $\delta$ (major isomer) 7.42-7.34 (m, 5H, Ar-H), 4.85 (dd, J 8.6, $4.7 \mathrm{~Hz}$, $1 \mathrm{H}, \mathrm{NCHPh}), 4.65-4.51\left(\mathrm{~m}, 2 \mathrm{H}, \mathrm{CHHCHPh}, \mathrm{NCHCH}_{2} \mathrm{PPr}\right), 4.20(\mathrm{dd}, J$ 8.6, $4.7 \mathrm{~Hz}, 1 \mathrm{H}$, $\mathrm{CH} H \mathrm{CHPh}), 3.89$ (m, 1H, $\left.\mathrm{CHCO}_{2}\right), 3.73$ (s, 6H, $\left.\mathrm{OCH}_{3}\right), 1.40-1.30$ (m, 1H, $\left.\mathrm{CHMe}_{2}\right), 1.15-1.05$ (m, 1H, CHHiPr), 0.90 (d, J $6.5 \mathrm{~Hz}, 3 \mathrm{H}, \mathrm{CH}_{3}$ ), 0.90-0.80 (m, 1H, CHHiPr), 0.33 (d, J 6.5 Hz, 3H, $\mathrm{CH}_{3}$ ) ppm. ${ }^{13} \mathrm{C}$ NMR (100 MHz, $\left.\mathrm{CDCl}_{3}\right): \delta$ (major isomer) $167.5\left(\mathrm{CO}_{2} \mathrm{Me}\right), 167.3\left(\mathrm{CO}_{2} \mathrm{Me}\right), 158.5$ $(\mathrm{C}=\mathrm{O}), 140.2,129.0(2 \mathrm{C}), 128.9(2 \mathrm{C}), 127.2,70.8\left(\mathrm{CH}_{2} \mathrm{CHPh}\right), 58.1\left(\mathrm{CHCH}_{2} \mathrm{iPr}\right), 55.1(\mathrm{CHPh})$, $52.9\left(\mathrm{CH}\left(\mathrm{CO}_{2} \mathrm{Me}\right)_{2}\right), 52.8\left(\mathrm{OCH}_{3}\right), 52.7\left(\mathrm{OCH}_{3}\right)$, 39.6, 26.8, 24.3, $20.8\left(\mathrm{CH}_{3}\right), 20.7\left(\mathrm{CH}_{3}\right)$ ppm. HRMS (ESI): calcd. for $\mathrm{C}_{19} \mathrm{H}_{26} \mathrm{NO}_{6}[\mathrm{M}+\mathrm{H}]^{+} 364.1760$; found 364.1774.

Dimethyl 2-\{2-methyl-1-[(R)-2-oxo-4-phenyloxazolidin-3-yl $]$ propyl\}malonate (3e). This compound was obtained by the General Procedure (see Table 1, entry 6). Yield: 60\%; 91\% de. Colorless oil. IR (film) $v_{\max }$ 3055, 2986, 2930, 2360, 2339, 2308, 1755 (C=O), 1436, 1419, 1265, 1216, 1161, 1048, 909, 738, $705 \mathrm{~cm}^{-1} .{ }^{1} \mathrm{H}$ NMR (400 MHz, $\mathrm{CDCl}_{3}$ ): $\delta$ (major isomer) 7.50-7.47 (m, 2H, Ar-H), 7.39-7.33 (m, 3H, Ar-H), 5.09 (dd, J 8.6, 4.0 Hz, 1H, NCHPh), $4.52(\mathrm{t}, J 8.6 \mathrm{~Hz}$, 1H, CHHOCO), 4.19 (dd, J 8.6, 4.0 Hz, 1H, CHHOCO), 4.10-4.03 (m, 1H, NCHiPr), 4.00 (d, J 7.1 $\left.\mathrm{Hz}, 1 \mathrm{H}, \mathrm{CHCO}_{2}\right), 3.72\left(\mathrm{~s}, 3 \mathrm{H}, \mathrm{OCH}_{3}\right), 3.70\left(\mathrm{~s}, 3 \mathrm{H}, \mathrm{OCH}_{3}\right), 1.95-1.85\left(\mathrm{~m}, 1 \mathrm{H}, \mathrm{CH}\left(\mathrm{CH}_{3}\right)_{2}\right), 0.77(\mathrm{~d}, J$ $\left.6.8 \mathrm{~Hz}, 3 \mathrm{H}, \mathrm{CHCH}_{3}\right), 0.65\left(\mathrm{~d}, J 6.6 \mathrm{~Hz}, 3 \mathrm{H}, \mathrm{CHCH}_{3}\right) \mathrm{ppm} .{ }^{13} \mathrm{C} \mathrm{NMR}\left(100 \mathrm{MHz}, \mathrm{CDCl}_{3}\right): \delta$ (major isomer) $168.4\left(\mathrm{CO}_{2} \mathrm{Me}\right), 167.9\left(\mathrm{CO}_{2} \mathrm{Me}\right), 158.7(\mathrm{C}=\mathrm{O}), 140.0,128.9(2 \mathrm{C}), 128.8(2 \mathrm{C}), 127.7,71.0$ $\left(\mathrm{CH}_{2} \mathrm{OCO}\right), 60.2(\mathrm{CH} i \mathrm{Pr}), 59.3(\mathrm{CHPh}), 53.3\left(\mathrm{CH}\left(\mathrm{CO}_{2} \mathrm{Et}\right)_{2}\right), 52.8\left(\mathrm{OCH}_{3}\right), 52.7\left(\mathrm{OCH}_{3}\right), 29.1$ $\left(\mathrm{CH}\left(\mathrm{CH}_{3}\right)_{2}\right), 19.9\left(\mathrm{CH}_{3}\right), 19.8\left(\mathrm{CH}_{3}\right)$ ppm. HRMS (ESI): calcd. for $\mathrm{C}_{18} \mathrm{H}_{24} \mathrm{NO}_{6}[\mathrm{M}+\mathrm{H}]^{+} 350.1604$; found 350.1590 .

Dimethyl 2-\{cyclohexyl[(R)-2-oxo-4-phenyloxazolidin-3-yl $]$ methyl $\}$ malonate $\quad(3 g)$ This compound was obtained by the General Procedure (see Table 1, entry 9). Yield: 21\%. 94\% de. Colorless oil. IR (film) $v_{\max }$ 3055, 2987, 2933, 2805, $1754(\mathrm{C}=\mathrm{O}), 1421,1265,1151,1056,896$, 738, $705 \mathrm{~cm}^{-1} .{ }^{1} \mathrm{H}$ NMR (400 MHz, $\mathrm{CDCl}_{3}$ ): $\delta$ (major isomer) 7.52-7.48 (m, 2H, Ar-H), 7.40-7.33 (m, 3H, Ar-H), 5.11 (dd, J 8.6, 3.7 Hz, 1H, NCHPh), 4.52 (t, $J 8.6 \mathrm{~Hz}, 1 \mathrm{H}, \mathrm{CHHOCO}), 4.24$ (dd, $J$ 8.6, 3.7 Hz, 1H, CHHOCO), 4.18-4.12 (m, 1H, NCHCy), 3.95 (d, J 6.9 Hz, 1H, $\left.\mathrm{CHCO}_{2}\right), 3.74$ (s, $\left.3 \mathrm{H}, \mathrm{OCH}_{3}\right), 3.73\left(\mathrm{~s}, 3 \mathrm{H}, \mathrm{OCH}_{3}\right), 1.78-1.64(\mathrm{~m}, 5 \mathrm{H}, \mathrm{Cy}-\mathrm{H}), 1.34-1.11(\mathrm{~m}, 5 \mathrm{H}, \mathrm{Cy}-\mathrm{H}), 1.50-1.35(\mathrm{~m}$, $1 \mathrm{H}, \mathrm{Cy}-\mathrm{H}) \mathrm{ppm} .{ }^{13} \mathrm{C} \mathrm{NMR}\left(100 \mathrm{MHz}, \mathrm{CDCl}_{3}\right): \delta$ (major isomer) $168.7(\mathrm{C}=\mathrm{O}), 168.2(\mathrm{C}=\mathrm{O}), 158.8$ $(\mathrm{C}=\mathrm{O}), \quad 140.4,128.8,128.8,127.8,71.1$ ( $\left.\mathrm{CH}_{2} \mathrm{OCO}\right), 59.2$ (CHNPh), 59.2 (CHCy), 53.2 $\left(\mathrm{CH}\left(\mathrm{CO}_{2} \mathrm{Me}\right)_{2}\right), 52.9\left(\mathrm{OCH}_{3}\right), 52.8\left(\mathrm{OCH}_{3}\right), 38.3,30.8,30.0,26.9,25.9,25.3$ ppm. HRMS (ESI): calcd. for $\mathrm{C}_{21} \mathrm{H}_{28} \mathrm{NO}_{6}[\mathrm{M}+\mathrm{H}]^{+} 390.1917$; found 390.1923.

Dimethyl 2-\{[(R)-2-oxo-4-phenyloxazolidin-3-yl $]$ (phenyl)methyl $\}$ malonate $\quad$ (3h). This compound was obtained by the General Procedure (see Table 1, entry 12). Yield: 70\%. 90\% de. Colorless solid; after recrystallization the major isomer was obtained as colorless crystals; mp 110 ${ }^{\circ} \mathrm{C}\left(\mathrm{Et}_{2} \mathrm{O}\right)$. IR (film) $v_{\max }$ 3055, 2987, $1760(\mathrm{C}=\mathrm{O}), 1421,1265,896,738,704 \mathrm{~cm}^{-1}$. ${ }^{1} \mathrm{H}$ NMR $(400$ $\mathrm{MHz}, \mathrm{CDCl}_{3}$ ): $\delta$ (major isomer) 7.46-7.39 (m, 3H, Ar H), 7.36-7.23 (m, 7H, Ar H), $5.30(\mathrm{~d}, J 12.0$ $\left.\mathrm{Hz}, 1 \mathrm{H}, \mathrm{CHCHCO}_{2}\right), 4.59$ (d, J $\left.12.0 \mathrm{~Hz}, 1 \mathrm{H}, \mathrm{CHCO}_{2}\right), 4.55-4.40$ (m, 2H, CHHOCO, $\left.\mathrm{NCHCH}_{2} \mathrm{O}\right)$, 4.05 (t, J $7.4 \mathrm{~Hz}, 1 \mathrm{H}, \mathrm{CH} H \mathrm{OCO}), 3.80\left(\mathrm{~s}, 3 \mathrm{H}, \mathrm{OCH}_{3}\right), 3.38\left(\mathrm{~s}, 3 \mathrm{H}, \mathrm{OCH}_{3}\right) \mathrm{ppm} .{ }^{13} \mathrm{C} \mathrm{NMR}(100$ $\left.\mathrm{MHz}, \mathrm{CDCl}_{3}\right): \delta$ (major isomer) $167.4(\mathrm{C}=\mathrm{O}), 167.4(\mathrm{C}=\mathrm{O}), 158.0(\mathrm{C}=\mathrm{O}), 136.2,135.9,129.5$, 
129.3, 129.0, 128.9, 128.3, 128.2, $70.2\left(\mathrm{CH}_{2} \mathrm{OCO}\right), 60.9\left(\mathrm{CH}_{2} \mathrm{CH}_{2} \mathrm{OCO}\right), 57.6\left(\mathrm{CHCH}\left(\mathrm{CO}_{2} \mathrm{Me}\right)_{2}\right)$, $53.6\left(\mathrm{CH}\left(\mathrm{CO}_{2} \mathrm{Me}\right)_{2}\right), 53.0\left(\mathrm{OCH}_{3}\right), 52.6\left(\mathrm{OCH}_{3}\right)$ ppm. HRMS (ESI): calcd. for $\mathrm{C}_{21} \mathrm{H}_{22} \mathrm{NO}_{6}[\mathrm{M}+$ $\mathrm{H}]^{+}$384.1447; found 384.1438 .

(S)-4-Methyl-3-\{(R)-2-oxo-4-phenyloxazolidin-3-yl $\}$ pentanoic acid (4). An aqueous solution of 6 $\mathrm{N} \mathrm{HCl}(7.5 \mathrm{~mL})$ was added to dimethyl 2-\{2-methyl-1-[(R)-2-oxo-4-phenyloxazolidin-3-yl]propyl $\}$ malonate $(3 \mathrm{e})(459 \mathrm{mg}, 1.31 \mathrm{mmol})$. The reaction mixture was heated at $100{ }^{\circ} \mathrm{C}$ for $15 \mathrm{~h}$. After cooling to room temperature, the aqueous phase was extracted with methylene chloride $(3 \times$ $10 \mathrm{~mL})$. The combined organic phases were washed with water $(2 \times 5 \mathrm{~mL})$, dried $\left(\mathrm{MgSO}_{4}\right)$, filtered and concentrated under vacuum afforded $294 \mathrm{mg}$ of crude compound. After two recrystallizations the carboxylic acid 4 was obtained as colorless crystals (198 mg). Yield: 53\%, 93\% de. mp 137-140 ${ }^{\circ} \mathrm{C}\left(\mathrm{Et}_{2} \mathrm{O}\right) .[\alpha]_{\mathrm{D}}^{20}+31.2\left(c 1.00, \mathrm{CH}_{2} \mathrm{Cl}_{2}\right)$. IR (KBr pellet) $v_{\max } 3160,2991,2955,2938,2871,1746$, 1731, 1703 (C=O), 1491, 1462, 1429, 1380, 1380, 1337, 1265, 944, 855, 773, 750, $705 \mathrm{~cm}^{-1} .{ }^{1} \mathrm{H}$ NMR (400 MHz, CDCl $): \delta 7.39$ (s, $5 \mathrm{H}, \mathrm{Ar} H$ ), 4.85 (dd, $J 8.9,6.6 \mathrm{~Hz}, 1 \mathrm{H}, \mathrm{NCHPh}), 4.63$ (t, $J 8.9$ $\mathrm{Hz}, 1 \mathrm{H}, \mathrm{CHHOCO}$ ), 4.24 (dd, J 8.9, $6.6 \mathrm{~Hz}, 1 \mathrm{H}, \mathrm{CH} H \mathrm{OCO}$ ), 3.60-3.50 (m, 1H, NCHiPr), 2.83 (dd, $\left.J 15.8,9.6 \mathrm{~Hz}, 1 \mathrm{H}, \mathrm{CHHCO}_{2} \mathrm{H}\right), 2.72\left(\mathrm{dd}, J 15.8,4.7 \mathrm{~Hz}, 1 \mathrm{H}, \mathrm{CH} H \mathrm{CO}_{2} \mathrm{H}\right), 1.72-1.60(\mathrm{~m}, 1 \mathrm{H}$, $\left.\mathrm{CH}\left(\mathrm{CH}_{3}\right)_{2}\right), 0.90\left(\mathrm{~d}, J 6.5 \mathrm{~Hz}, 3 \mathrm{H}, \mathrm{CH}_{3}\right), 0.83\left(\mathrm{~d}, J 6.5 \mathrm{~Hz}, 3 \mathrm{H}, \mathrm{CH}_{3}\right) \mathrm{ppm} .{ }^{13} \mathrm{C} \mathrm{NMR}(100 \mathrm{MHz}$, $\left.\mathrm{CDCl}_{3}\right): \delta 175.9\left(\mathrm{CO}_{2} \mathrm{H}\right), 158.5(\mathrm{C}=\mathrm{O}), 138.4,129.2,129.1(2 \mathrm{C}), 127.7(2 \mathrm{C}), 70.2\left(\mathrm{CH}_{2} \mathrm{OCO}\right), 60.3$ $(\mathrm{CH} i \mathrm{Pr}), 58.0(\mathrm{CHPh}), 34.7\left(\mathrm{CH}_{2} \mathrm{CO}_{2} \mathrm{H}\right), 30.9\left(\mathrm{CHCH}_{3}\right), 20.0\left(\mathrm{CH}_{3}\right), 19.4\left(\mathrm{CH}_{3}\right)$ ppm. HRMS (ESI): calcd. for $\mathrm{C}_{15} \mathrm{H}_{20} \mathrm{NO}_{4}[\mathrm{M}+\mathrm{H}]^{+}$278.1392; found 278.1385.

(S)-3-Amino-4-methylpentanoic acid (5). A solution of 4-methyl-3-((R)-2-oxo-4-phenyloxazolidin-3-yl)pentanoic acid (4) $(51.2 \mathrm{mg}, 0.185 \mathrm{mmol})$ in THF $(6.5 \mathrm{~mL})$ and $t$-BuOH $(0.185$ $\mathrm{mL})$ was cooled under argon at $0{ }^{\circ} \mathrm{C}$. Lithium $(13.2 \mathrm{mg}, 10$ equiv) was placed under argon in another flask equipped with a dry-ice condenser. After cooling at $-78^{\circ} \mathrm{C}, \mathrm{NH}_{3}(13 \mathrm{~mL})$ was flushed in. After $10 \mathrm{~min}$, the solution of oxazolidinone 4 was added dropwise to the deep blue solution obtained. After stirring at $-78{ }^{\circ} \mathrm{C}$ for $30 \mathrm{~min}$, powdered $\mathrm{NH}_{4} \mathrm{Cl}(0.17 \mathrm{~g})$ was added. The flask was allowed to warm to room temperature under a stream of nitrogen, which facilitated the elimination of ammonia. After concentration under vacuum, the residue obtained was purified using a DOWEX 50WX42400 ion-exchange resin washed before-hand with water. The resin was eluted successively with water, then $0.1 \mathrm{M}, 0.5 \mathrm{M}$ and $1 \mathrm{M}$ aqueous $\mathrm{NH}_{4} \mathrm{OH}$. The content of each fraction was checked by thin-layer chromatography (ninhydrin). After concentration of the relevant fractions, (S)-3amino-4-methylpentanoic acid (5) was obtained as a colorless solid $(18.6 \mathrm{mg})$. Yield: $77 \%$. Colorless powder; mp 202-205 ${ }^{\circ} \mathrm{C}$, lit.., ${ }^{5} 197-198{ }^{\circ} \mathrm{C}$. $[\alpha]_{\mathrm{D}}{ }^{20}-49.6$ (c 0.4, water), lit., ${ }^{5}-53$ (c 2.0, water). IR (KBr pellet) $v_{\max } 2965,2355,2131,1728,1712,1554,1535,1468,1390,1324,1246$, 1118, 1037, $708 \mathrm{~cm}^{-1} .{ }^{1} \mathrm{H}$ NMR $\left(400 \mathrm{MHz}, \mathrm{D}_{2} \mathrm{O}\right): \delta 3.33(\mathrm{~m}, 1 \mathrm{H}, \mathrm{CHN}), 2.58(\mathrm{dd}, J 16.8,4.2 \mathrm{~Hz}$, $\left.1 \mathrm{H}, \mathrm{C} H \mathrm{HCO}_{2} \mathrm{H}\right), 2.40\left(\mathrm{dd}, J 16.8,9.3 \mathrm{~Hz}, 1 \mathrm{H}, \mathrm{CH} H C O_{2} \mathrm{H}\right), 1.95\left(\mathrm{~m}, 1 \mathrm{H}, \mathrm{CHCH}_{3}\right), 1.00(\mathrm{~d}, J 6.5$ $\left.\mathrm{Hz}, 3 \mathrm{H}, \mathrm{CH}_{3}\right), 0.98\left(\mathrm{~d}, J 6.5 \mathrm{~Hz}, 3 \mathrm{H}, \mathrm{CH}_{3}\right) \mathrm{ppm} .{ }^{13} \mathrm{C} \mathrm{NMR}\left(100 \mathrm{MHz}, \mathrm{D}_{2} \mathrm{O}\right): \delta 178.5\left(\mathrm{CO}_{2} \mathrm{H}\right), 54.8$ (CHiPr), $35.9\left(\mathrm{CH}_{2} \mathrm{CO}_{2} \mathrm{H}\right), 30.0\left(\mathrm{CHCH}_{3}\right), 17.4\left(\mathrm{CH}_{3}\right), 17.2\left(\mathrm{CH}_{3}\right) \mathrm{ppm}$.

Crystallography. The data were collected at 150(2) K on a Nonius Kappa-CCD area detector diffractometer ${ }^{31}$ using graphite-monochromated Mo-Ka radiation $(\lambda 0.71073 \AA)$, and they were 
processed with HKL2000. ${ }^{32}$ No absorption correction was done. The structure was solved by direct methods with SHELXS-97 and refined by full-matrix least-squares on $F^{2}$ with SHELXL-97. ${ }^{33}$ All non-hydrogen atoms were refined with anisotropic displacement parameters. In the absence of a suitable anomalous scatterer, the Friedel pairs have been merged, the absolute configuration of the unknown chiral centre being deduced from that of the known one. Crystal data for $\mathbf{3 h}$ (major isomer): $\mathrm{C}_{21} \mathrm{H}_{21} \mathrm{NO}_{6}, M=383.39$, orthorhombic, space group $P 2_{1} 2_{1} 2_{1}, a=6.9024(4), b=$ 14.8607(14), $c=18.8527(19) \AA, V=1933.8(3) \AA^{3}, Z=4$. Refinement of 255 parameters on 2122 independent reflections out of 50852 measured reflections $\left(R_{\mathrm{int}}=0.031\right)$ led to $R 1=0.044, w R 2=$ $0.099, \Delta \rho_{\min }=-0.22, \Delta \rho_{\max }=0.15 \mathrm{e} \AA^{-3}$. CCDC-990814 contains the supplementary crystallographic data for this paper. These data can be obtained free of charge from The Cambridge Crystallographic Data Centre via www.ccdc.cam.ac.uk/data_request/cif.

\section{Acknowledgements}

We thank the Ministère de la recherche et de l'enseignement supérieur (doctoral grant to H. H.).

\section{References}

1. Cardillo, G.; Tomasini, C. Chem. Soc. Rev. 1996, 25, 117-128.

2. Enantioselective Synthesis of $\beta$-Amino Acids; Juaristi, E.; Soloshonok, V., Eds. John Wiley \& Sons Inc.: Hoboken, 2005.

3. Weiner, B.; Szymański, W.; Janssen, D. B.; Minnaard, A. J.; Feringa, B. L. Chem. Soc. Rev. 2010, 39, 1656-1691.

http://dx.doi.org/10.1039/b919599h

4. Davies, S. G.; Smith, A. D.; Price, P. D. Tetrahedron: Asymmetry 2005, 16, 2833-2891 and references cited therein.

5. Davies, S. G.; Fletcher, A. M.; Roberts, P. M. Org. Synth. 2010, 87, 143-160.

6. Bentley, S. A.; Davies, S. G.; Lee, J. A.; Roberts, P. M.; Thomson, J. E. Org. Lett. 2011, 13, 2544-2547. http://dx.doi.org/10.1021/ol200625h

7. Etxebarria, J.; Vicario, J. L.; Badia, D.; Carrillo, L.; Ruiz, N. J. Org. Chem. 2005, 70, 8790-8800. http://dx.doi.org/10.1021/jo051207j

8. Sani, M.; Bruche, L.; Chiva, G.; Fustero, S.; Piera, J.; Volonterio, A.; Zanda, M. Angew. Chem., Int. Ed. 2003, 42, 2060-2063. http://dx.doi.org/10.1002/anie.200250711

9. Dumas, F.; Mezrhab, B.; d'Angelo, J. J. Org. Chem. 1996, 61, 2293-2304. http://dx.doi.org/10.1021/jo951414r

10. Shida, N.; Kabuto, C.; Niwa, T.; Ebata, T.; Yamamoto, Y. J. Org. Chem. 1994, 59, 4068-4075. http://dx.doi.org/10.1021/jo00094a013 
11. Amoroso, R.; Cardillo, G.; Sabatino, P.; Tomasini, C.; Trerè, A. J. Org. Chem. 1993, 58, 5615-5619. http://dx.doi.org/10.1021/jo00073a017

12. Sakai, T.; Kawamoto, Y.; Tomioka, K. J. Org. Chem, 2006, 71, 4706-4709. http://dx.doi.org/10.1021/jo060588d

13. Suzuki, M.; Kawamoto, Y.; Sakai, T.; Yamamoto, Y.; Tomioka, K. Org. Lett. 2009, 11, 653-655. http://dx.doi.org/10.1021/o1802759j

14. Cardillo, G.; Gentilucci, L.; Gianotti, M.; Kim, H.; Perciaccante, R.; Tolomelli, A. Tetrahedron: Asymmetry 2001, 12, 2395-2398. http://dx.doi.org/10.1016/S0957-4166(01)00427-X

15. Zhuang, W.; Hazell, R. G.; Jørgensen, K. A. Chem. Commun. 2001, 1240-1241.

16. Sibi, M. P.; Shay, J.J.; Liu, M.; Jasperse, C. P. J. Am. Chem. Soc. 1998, 120, 6615-6616. http://dx.doi.org/10.1021/ja980520i

17. Lucet, D.; Toupet, L.; Le Gall, T.; Mioskowski, C. J. Org. Chem. 1997, 62, 2682-2683. http://dx.doi.org/10.1021/jo970390g

18. Lucet, D.; Sabelle, S.; Kostelitz, O.; Le Gall, T.; Mioskowski, C. Eur. J. Org. Chem. 1999, 2583-2591.

19. Leroux, M.-L.; Le Gall, T.; Mioskowski, C. Tetrahedron: Asymmetry 2001, 12, 1817-1823.

20. Yeom, C.-E.; Kim, M. J.; Kim, B. M. Tetrahedron 2007, 63, 904-909. http://dx.doi.org/10.1016/j.tet.2006.11.037

21. Ménand, M.; Dalla, V. Synlett 2005, 95-98.

22. Cardillo, G.; Gentilucci, L.; Gianotti, M.; Perciaccante, R.; Tolomelli, A. J. Org. Chem. 2001, 66, 86578660. http://dx.doi.org/10.1021/jo015907+

23. Cardillo, G.; Fabbroni, S.; Gentilucci, L.; Gianotti, M.; Tolomelli, A. Synth. Commun. 2003, 33, 15871594. http://dx.doi.org/10.1081/SCC-120018782

24. Ranslow, P. B. D.; Hegedus, L. S.; de los Rios, C. J. Org. Chem. 2004, 69, 105-111.

25. Shin, D.-Y.; Jung, J.-K.; Seo, S.-Y.; Lee, Y.-S.; Paek, S.-M.; Chung, Y. K.; Shin, D. M.; Suh, Y.-G. Org. Lett. 2003, 5, 3635-3638. http://dx.doi.org/10.1021/o1035289e

26. Marianacci, O.; Micheletti, G.; Bernardi, L.; Fini, F.; Fochi, M.; Pettersen, D.; Sgarzani, V.; Ricci, A. Chem. Eur. J. 2007, 13, 8338-8351. http://dx.doi.org/10.1002/chem.200700908

27. Evans, D. A.; Sjogren, E. B. Tetrahedron Lett. 1985, 26, 3783-3786. http://dx.doi.org/10.1016/S0040-4039(00)89250-3

28. Colson, P.-J.; Hegedus, L. S. J. Org. Chem. 1993, 58, 5918-5924. http://dx.doi.org/10.1021/jo00074a016

29. Turconi, J.; Lebeau, L.; Paris, J. M.; Mioskowski, C. Tetrahedron 2006, 62, 8109-8114. http://dx.doi.org/10.1016/j.tet.2006.06.013

30. Sreejith, S. P.; Sani, M.; Terraneo, G.; Zanda, M. Synlett 2009, 1341-1345.

31. Hooft, R. W. W. COLLECT; Nonius BV: Delft, The Netherlands, 1998. 
32. Otwinowski, Z.; Minor, W. Methods Enzymol. 1997, 276, 307-326.

33. Sheldrick, G. M. Acta Crystallogr. Sect. A 2008, 64, 112-122.

\section{Graphical Abstract}

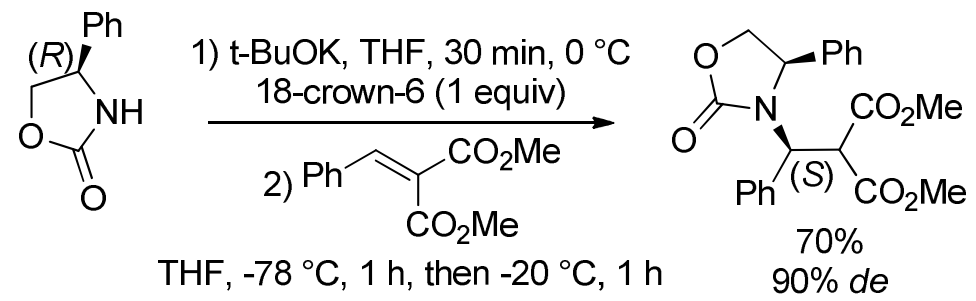

